\title{
Direct visualization of transcriptional activation by physical enhancer-promoter proximity
}

\author{
Hongtao Chen $^{a}$, Miki Fujioka ${ }^{b}$, James B. Jaynes ${ }^{b}$, and Thomas Gregor ${ }^{a *}$ \\ ${ }^{a}$ Joseph Henry Laboratories of Physics, and the Lewis-Sigler Institute for \\ Integrative Genomics, Princeton University, Princeton NJ 08544, USA \\ ${ }^{b}$ Department of Biochemistry and Molecular Biology, and the Kimmel Cancer Center, \\ Thomas Jefferson University, Philadelphia PA 1910\%, USA
}

(Dated: December 30, 2016)

\begin{abstract}
A long-standing question in metazoan gene regulation is how remote enhancers communicate with their target promoters over long distances. Combining genome editing and quantitative live imaging we simultaneously visualize physical enhancer-promoter communication and transcription in Drosophila embryos. Enhancers regulating pair rule stripes of even-skipped expression activate transcription of a reporter gene over a distance of $150 \mathrm{~kb}$. We show in individual cells that activation only occurs after the enhancer comes into close proximity with its regulatory target and that upon dissociation transcription ceases almost immediately. We further observe distinct topological conformations of the eve locus, depending on the spatial identity of the activating stripe enhancer. In addition, long-range activation results in transcriptional competition at the endogenous eve locus, causing corresponding developmental defects. Overall, we demonstrate that sustained physical proximity and enhancer-promoter engagement are required for enhancer action, and we provide a path to probe the implications of long-range regulation on cellular fates.
\end{abstract}

\section{INTRODUCTION}

Enhancers play a key role in the control of gene expression and development (Benoist and Chambon, 1981; Levine, 2010; Long et al., 2016). These 50-1500 base pair cis-regulatory elements stimulate transcription from core promoters in a time and tissue specific manner by recruiting context-dependent transcriptional activators and repressors (Levine, 2010; Buecker and Wysocka, 2012). Functions of enhancers are thought to be independent of their position and orientation. Enhancers can act in cis and in trans (Lee and Wu, 2006; Markenscoff-Papadimitriou et al., 2014), can be located upstream or downstream of the core promoter as well as in introns, and as far as a Mb from the promoter (Long et al., 2016). Whole-genome methods have shown that the human genome is riddled with enhancers, with estimates ranging from 200,000 to over a million (Consortium, 2012). Importantly, a significant fraction of these enhancers are located at large genomic distances from the promoters they regulate (Tolhuis et al., 2002; Uslu et al., 2014; Zhang et al., 2013). Even for a compact genome like Drosophila melanogaster, at least $30 \%$ of enhancerpromoter interactions occur over $20 \mathrm{~kb}$, and in many cases over intervening genes (Arnold et al., 2013; Ghavi-Helm et al., 2014; Kvon et al., 2014).

In this paper we focus on the mechanism by which enhancers communicate with their target genes over large distances. Several models have been proposed (Benabdallah and Bickmore, 2015; Blackwood and Kadonaga, 1998), the most popular of which involves looping of the flexible chromatin polymer such that distal enhancers come into direct contact with their promoter targets. Early evidence from genetic experiments has already revealed the presence of functional long-range enhancer-promoter communication in both vertebrates and flies (Lettice et al., 2003; Lewis,

*Correspondence: tg2@princeton.edu
1978; Qian et al., 1993; Sagai et al., 2005). Recently, chromatin conformation capture (3C) based approaches, involving the cross-linking of chromatin, revealed the physical proximity of distal enhancers and promoters in a variety of vertebrate cells and tissues (Kagey et al., 2010; Mifsud et al., 2015; Spitz, 2016). Yet, despite these demonstrations of both functional and physical interactions, fundamental questions about how enhancers communicate with their target promoters over large distances and how they activate transcription still remain (Levine et al., 2014).

A major gap in our understanding pertains to the role of physical enhancer-promoter interactions in transcriptional regulation. On the one hand, 3C-based experiments have revealed extensive enhancer-promoter interactions that are conserved among developmental stages, cell fates or evolution, suggesting a permissive role of the physical enhancerpromoter interactions on transcriptional activity (GhaviHelm et al., 2014; Jin et al., 2013; Sanyal et al., 2012). On the other hand, lineage-specific enhancer-promoter interactions are found to be prevalent in many developmental contexts, arguing for the possibility of an instructive role (Dixon et al., 2015; Javierre et al., 2016). Part of the reason for this discrepancy lies in the fact that the static picture gained from currently employed techniques can only provide correlative evidence. Furthermore, most genomic experiments rely on bulk assays, which might cover temporal and spatial heterogeneity within the samples.

Crucially, a direct dynamic link between enhancerpromoter proximity and transcriptional activation is lacking. Is proximity needed for transcriptional activation, or is it a consequence of transcriptional activity? Even if the most popular hypothesis is that proximity is necessary for transcriptional activation, the above discussed studies either measure transcriptional activity or physical proximity, and experiments that measure both transcription and physical proximity simultaneously have not been reported. What degree of physical proximity is needed for activation? Is there a topological distinction at the gene locus when different enhancers are activating the gene? It is 
further unclear whether physical proximity is needed transiently for a limited time interval to establish an active state - a state that can be remembered once proximity is lost - or whether continued transcription requires that the enhancer and promoter remain in sustained proximity. To address these fundamental questions an approach that simultaneously captures the dynamics of enhancer-promoter distances and the dynamics of transcriptional activity is necessary.

Here we have devised an assay that uses a combination of genome editing, genetics, and live single-cell imaging to visualize the relationship between enhancer activation of transcription and physical proximity in real time. We show that activation of transcription can only occur when the enhancer comes into close proximity with its regulatory target and that transcription ceases almost immediately when they move apart. Moreover, we find that proximity alone is not sufficient for enhancer action; rather enhancer action requires a direct engagement of the enhancer with its target promoter. Our results suggest that establishment of physical interactions between enhancers and promoters can be the key rate-limiting step in gene regulation over long distances, and we can exclude mechanisms of transient enhancer-promoter associations leading to stable transcriptional activity.

\section{RESULTS}

\section{Genetic Design: Homie-dependent long-distance regulation}

To determine whether physical proximity is central to enhancer-promoter communication we have taken advantage of a characteristic property of boundary (insulator) elements in flies, namely their ability to pair with themselves, often over large genomic distances. For this purpose we selected a boundary called, homie, which marks the 3 end of the even-skipped (eve) locus (Fujioka et al., 2013; Fujioka et al., 2009). Genetic studies have shown that homie-homie self-pairing interactions can orchestrate enhancer activation of a reporter at distances of at least 2 Mb (Fujioka et al., 2013).

In our experimental system a transgene consisting of the eve promoter (no enhancers) and the lac $Z$ coding sequence is inserted at an attP site located $142 \mathrm{~kb}$ upstream of the eve gene (Fig. 1A). When homie is included in this reporter transgene, physical interactions between it and the homie boundary at the endogenous eve locus can be detected in chromosome conformation capture experiments (Fujioka et al., 2016). In fixed embryos sporadic expression of lac $Z$ mRNA is observed solely within the limits of the endogenous eve stripes (Fig. 1B and 1C). These findings indicate that the activation of the lac $Z$ reporter depends on the enhancers in the eve locus $142 \mathrm{~kb}$ away, and that at this stage in development, the promoter of the reporter has no spontaneous activity nor does it respond to enhancers near the site of insertion.
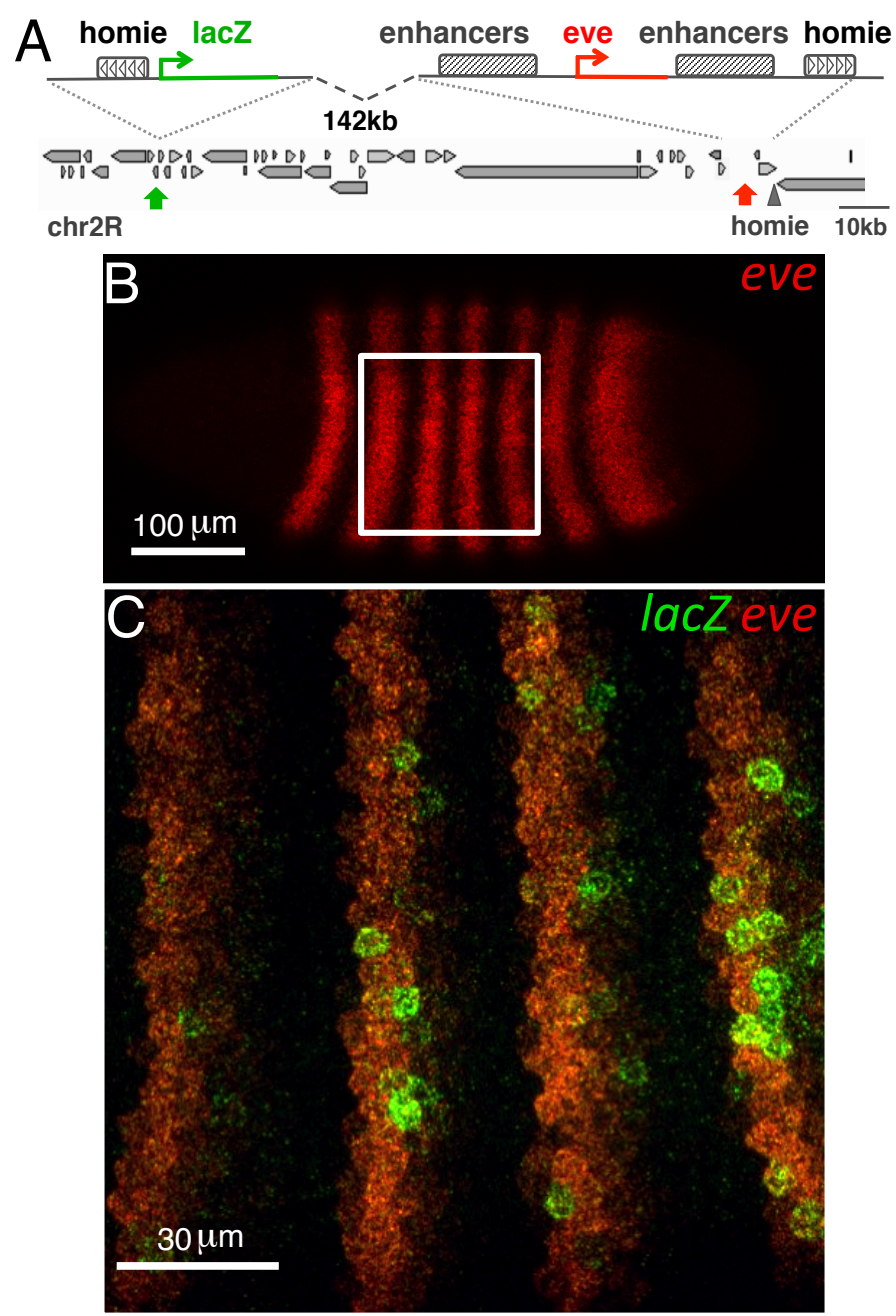

FIG. 1: An endogenous genomic construct is designed to investigate long-distance enhancer-promoter interactions. (A) Scheme of the construct design and its genomic context. Ectopic homie insulator sequence with an eve promoter driving lac $Z$ is integrated at $-142 \mathrm{~kb}$ upstream of the endogenous eve locus in the Drosophila genome. (B) Upper panel: surface view of a $2.5 \mathrm{~h}$ old Drosophila embryo hybridized with red fluorescent eve mRNA probes. Lower panel: z-stack projection of the marked region in the upper panel. LacZ activity (revealed using green fluorescent lac $Z$ mRNA hybridization probes) only occurs sporadically within the limits of the eve pattern (red).

\section{Visualization of transcription and enhancer-promoter dynamics}

Key to exploring the connection between enhancer action and physical enhancer-promoter proximity is being able to simultaneously visualize the location of the promoter, the location of the enhancers, and the transcriptional activity in living embryos. For this purpose we introduced tags into the endogenous eve gene and the $l a c Z$ reporter transgene at $-142 \mathrm{~kb}$ (Fig. 1A). First, we used genome editing to insert an MS2 stem loop cassette (Bertrand et al., 1998; Larson et al., 2011; Yunger et al., 2010) into the 1st intron of the eve gene. Maternally expressed MS2 coat protein (MCP) fused to a blue fluorescent protein was used to visualize not only nascent eve transcripts but also mark the 


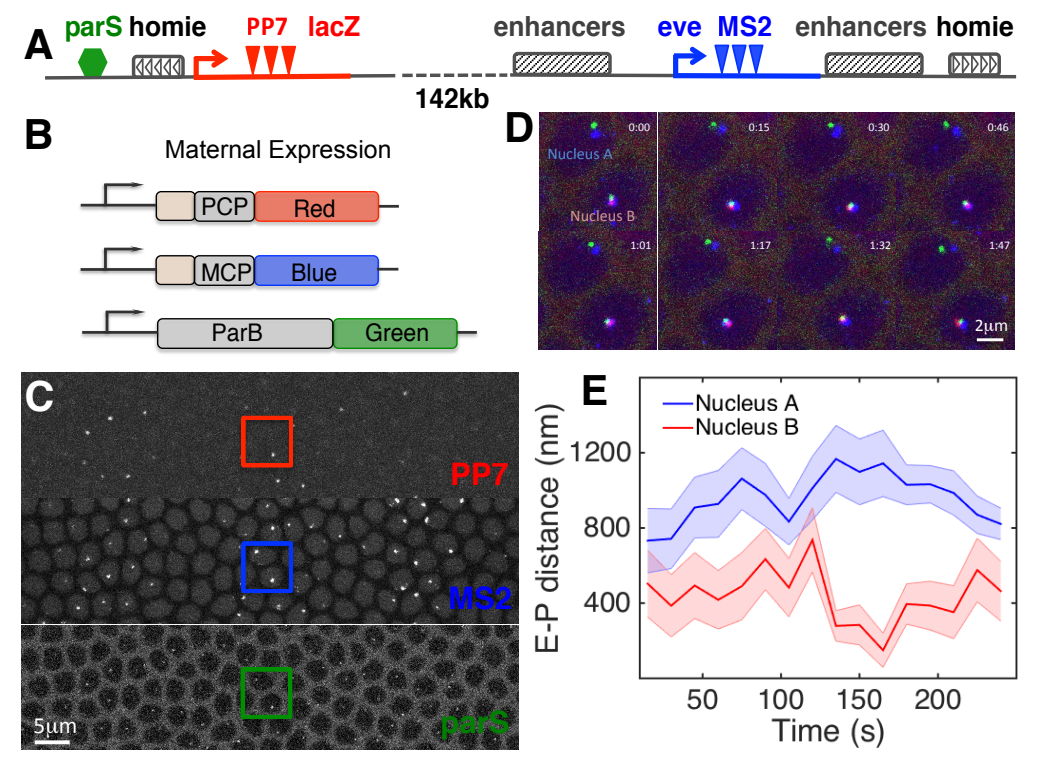

FIG. 2: Visualization of enhancer-promoter movements producing transcriptional activity using three-color live imaging. (A) Editing the endogenous eve locus. 24xMS2 stem loops are knocked into the 1st intron of eve to measure eve transcriptional activity as well as the position of the locus. $24 \mathrm{xPP} 7$ stem loops are inserted into the homie-lac $Z$ transgene to monitor lacZ transcription, and a parS tag is introduced next to the ectopic homie sequence for monitoring the movement of the reporter locus at $-142 \mathrm{~kb}$. (B) Design of genetic constructs: PCP::red, MCP::blue, and ParB::green fusion proteins that bind PP7 and MS2 stem loops, and parS sequences, respectively, are provided maternally. (C) Snapshot of an F1 embryo from females carrying all fluorescent proteins in (B), mated with males carrying the genomic construct shown in (A). The embryo displays fluorescent foci for MS2 (blue), PP7 (red) and parS (green) in the corresponding channels. (D) 8 snapshots of a time course following two nuclei for 2 min. Nuclei A and B have eve activity (blue); nucleus B also has lac $Z$ activity (red). (E) Instantaneous enhancer-promoter (E-P) distance between endogenous eve enhancers (as measured by blue signal) and the transgenic reporter (as measured by green signal) as a function of time for nuclei A (blue) and B (red) from (D). Error bar corresponds to measurement error. See also Fig. S1 and Fig. S2.

nuclear location of the eve gene and the associated eve enhancers. Second, to visualize transcriptional activity of the lacZ reporter we placed a PP7 stem loop cassette (Fukaya et al., 2016; Hocine et al., 2013) near the 5 end of the lacZ coding sequence. Nascent transcripts expressed from the reporter can then be visualized by the binding of maternally expressed PP7 coat proteins (PCP) fused to a red fluorescent protein (Fig. 2A). Finally, to mark the position of the lac $Z$ reporter independent of whether the reporter is active, we used the recently developed parS/parB DNA labeling system (Dubarry et al., 2006; Saad et al., 2014). This system employs Burkholderia parS DNA sequences that nucleate the binding of a ParB-GFP fusion proteins, and it is thought to be less disruptive to the local chromatin structure than more traditional DNA labeling systems (Bystricky, 2015).

Using three-color time-lapse confocal microscopy we captured stacks of optical sections of the surface of two-hours old embryos carrying the tagged eve locus and the parShomie-lacZ reporter. In these stacks we can clearly identify individual fluorescent foci in 70-100 nuclei simultaneously (Fig. 2B). In the blue channel we observed the endogenous activity and transcriptional dynamics of the eve gene in its characteristic seven-striped pattern. This pattern is quantitatively identical to that observed from the endogenous eve gene (Fig. S1). In the green channel we saw the parB foci in all of the nuclei in the embryo, which trace the movement of the lac $Z$ reporter within the nucleus. Finally, in the red channel we observed $l a c Z$ expression in a subset of nuclei in the (blue) eve stripes. Consistent with the results from our fixed embryos, lac $Z$ expression is restricted to nuclei that reside within one of the seven eve stripes.

To test our ability to reliably and accurately measure the localization of the reporter and the eve gene we generated a synthetic construct (localization control) in which all three fluorescent proteins are localized within a genomic distance of $2.0 \mathrm{~kb}$ (Fig. S2A). By analyzing embryos carrying this construct, we were able to calibrate chromatic aberrations originating from the microscope and also estimate errors in measuring spot localization (Fig. S2B-H). We also tested whether our method of marking the location of the reporter introduces perturbations in the chromatin structure that would hinder our analysis. For this purpose we placed the $\operatorname{par} S$ sequence at different locations relative to the lacZ reporter (Fig. S3A). We also implemented the more traditional $l a c O / \mathrm{LacI}$ system to mark the location of the reporter in the nucleus (Gasser, 2002; Sinclair et al., 2010). No significant difference in chromatin dynamics and transcription kinetics were observed when the parS tag was placed at different locations or replaced by the lacO tag (Fig. S3B-H). Based on these analyses, we conclude that our genomic labeling approach allows us to measure chromatin dynamics with an error of $180 \pm 6 \mathrm{~nm}$ (mean \pm SEM).

Our initial visualization of reporter activity revealed a close connection between transcription and physical proximity. In nuclei in which the reporter is inactive, the reporter is well separated from the eve gene. In this case the green focus, which marks the parS sequence in the re- 
porter, and the blue focus, which tags the transcriptionally active eve gene are far from each other, and since there is no red focus the reporter is silent (Fig. 2C). In nuclei in which the lacZ reporter is $\mathrm{ON}$, the parS-homie-lacZ transgene co-localizes with the eve gene. In these nuclei the blue, green and red fluorescent foci appear to be attached together (Fig. 2C). This difference is recovered in measurements of the instantaneous spatial distance between the blue and green foci. In nuclei where red reporter activity is present, the distance between the blue and green foci is significantly shorter than the blue-green distance in nuclei lacking the red focus throughout the interval of observation (Fig. 2D and Fig. S3B-C).

\section{Spatial proximity is necessary for enhancer action}

To provide a more detailed picture of how enhancer action is related to spatial proximity, we measured the physical separation between the eve gene (blue) and the lac Z reporter (green) in embryos carrying the experimental transgene, parS-homie-lacZ. We analyzed live images of 2528 nuclei across 35 individual embryos over a $30 \mathrm{~min}$ period in nuclear cycle 14 (Fig. 3A). We observed a bi-modal distribution for the time-averaged physical distance (rootmean-squared (RMS) distance, see Methods) that could be modeled as a mixture of two Gaussians (Fig. 3B) at $743 \pm 96$ $\mathrm{nm}$ and at $361 \pm 75 \mathrm{~nm}$, respectively (mean $\pm \mathrm{STD}$ ).

To confirm that the linkage of the reporter to eve is dependent on homie we replaced the homie sequence with lambda DNA of the same length. We generated a parSlambda-lacZ transgene and analyzed movies of 870 nuclei across 12 individual embryos, again for $30 \mathrm{~min}$ in nuclear cycle 14 (Fig. 3A, yellow). For this data set, the distribution of the MS2-parS (blue-green) RMS distance is unimodal (Fig. 3C) with a mean at $743 \pm 92 \mathrm{~nm}($ mean \pm STD). While the distance between this transgene and eve fluctuates during the $30 \mathrm{~min}$ interval, there is no instance in which sustained physical proximity is established.

When we further tested the effect of reversing the orientation of homie in the original transgene (Fujioka et al., 2016), such that the lac $Z$ reporter is downstream of homie, we find that pairing still occurs, but the regulation of the reporter by the eve enhancers is disrupted. We analyzed movies of 761 nuclei across 10 embryos for this parShomieR-lacZ transgene. As expected, the corresponding bi-modal distribution resembles that for the regular homie transgene and has two means at $739 \pm 104 \mathrm{~nm}$ and $334 \pm 63$ $\mathrm{nm}($ mean \pm STD), respectively (Fig. 3D).

We next scored the nuclei with respect to the transcriptional activity of the lacZ reporter. Strikingly, all of the parS-homie-lac $Z$ transgene nuclei showing lac $Z$ transcription (i.e. presence of red signal, $\mathrm{N}=192$ ) have a physical separation that falls within the distribution corresponding to the bound (i.e. homie-linked) conformation (Fig. $3 \mathrm{~A}$ and $3 \mathrm{~B})$. But there are no nuclei in the unbound conformation that also express lacZ. Taken together, the above results demonstrate that homie pairing creates a local chromatin conformation that is permissive to transcription events by ensuring physical proximity between the eve enhancers and the promoter of lacZ. Consistent with this
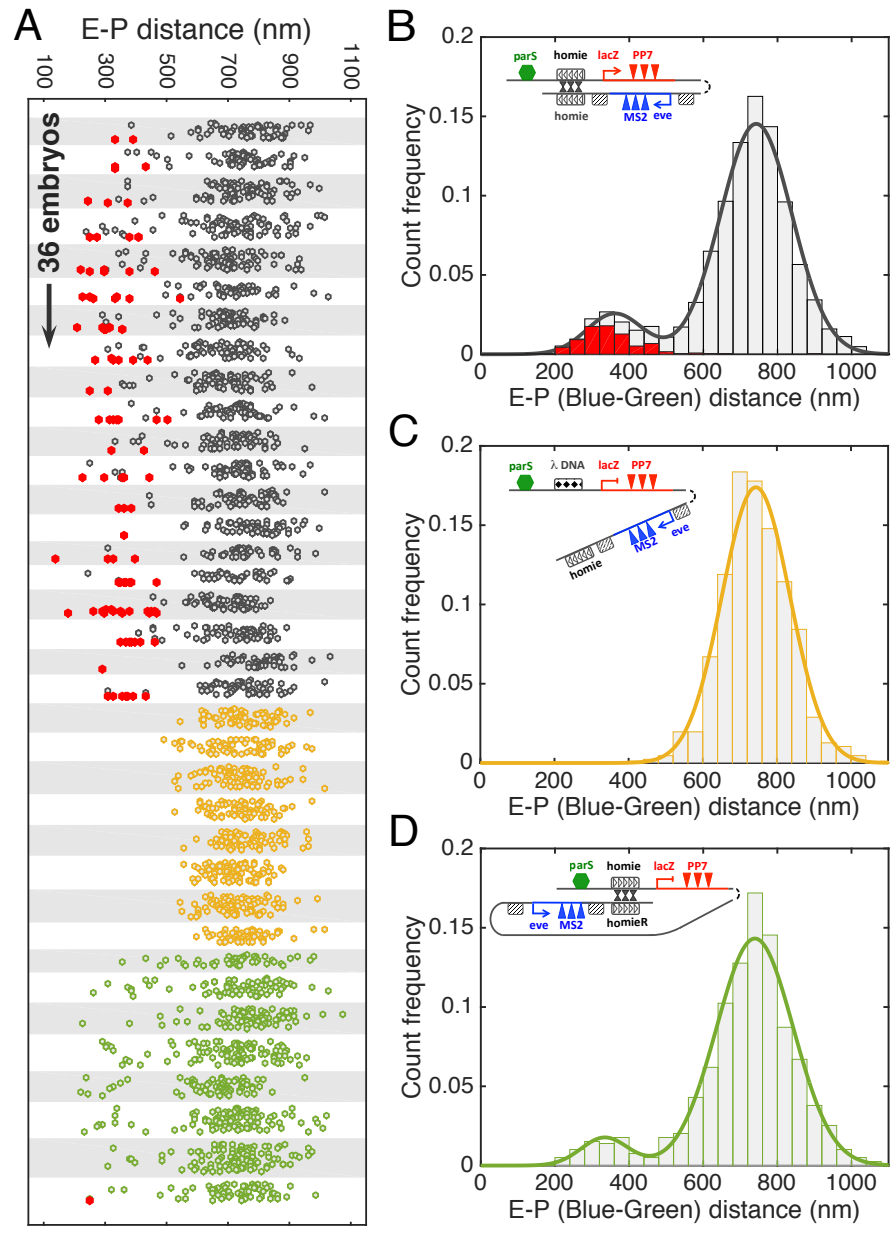

FIG. 3: Physical proximity between enhancers and promoter is required to activate transcription. (A) For 36 embryos during nuclear cycle 14 (arranged vertically) the distribution of the time-averaged root-mean-squared (RMS) distance (E-P distance) between blue MS2 and green parS DNA foci is depicted in vertical scatter plots. Each data point corresponds to an individual nucleus displaying eve activity in embryos expressing parS-homie-lacZ (gray), parS-lambda-lacZ (orange) and parS-homieR-lacZ (green), respectively. Nuclei displaying lac $Z$ activity are marked in red. (B-D) Distributions of RMS distances for three constructs: parS-homie-lacZ (B), parS-lambda-lacZ (C), parS-homieR-lacZ (D) with Gaussian fits. Insets show the genomic constructs in their presumptive conformations. See also Fig. S3.

view, none of the nuclei in control parS-lambda-lacZ embryos express lacZ. From these observations we conclude that the eve enhancers must be in close proximity to the lac $Z$ promoter in order to activate transcription.

\section{Necessity for sustained physical association}

To assess the temporal relationship between enhancerpromoter proximity and transcriptional activation, we examine the dynamics of physical proximity and activation along our measurement window, in singe nuclei. All nuclei displaying a switch from OFF-to- $\mathrm{ON}(\mathrm{N}=65)$ were aligned with respect to the time point when nascent transcripts could first be detected (Fig. 4A, see Methods). The data 
show a sharp switch in activity state with similar rates to those previously reported for nuclei exiting mitosis (Garcia et al., 2013). For the same data set, we then measured the mean distance between the green parS tag in the parSHomie-lacZ transgene and the eve gene (blue) as a function of time by averaging across all OFF-to-ON time courses with the same alignment (Fig. 4B). We observed a continuous spatial convergence until the onset of transcription at which point the mean distance corresponds to an average separation of about $340 \mathrm{~nm}$. These findings indicate that there is a close connection between the establishment of enhancer-promoter proximity and enhancer activation of transcription.

We also assessed the temporal relationship between the ON-to-OFF switch in transcription and the physical distance between the enhancer-promoter pair. In 42 nuclei that switch their transcriptional activity OFF during our observation window, a drop in transcriptional activity of the lac $Z$ reporter is accompanied by an increase in the mean distance between the parS-Homie-lacZ transgene and the eve gene (Figs. $4 \mathrm{C}$ and $4 \mathrm{D}$ ). Our experiments indicate that there is about a 4 min gap between the time when the parS-Homie-lacZ transgene first begins to detectably separate from the eve gene and a clear decline in transcriptional activity. Since our reporter gene is $5.5 \mathrm{~kb}$ in length and the measured RNA polymerase II (Pol II) elongation rate is $1.50 .1 \mathrm{~kb} / \mathrm{min}$ (Garcia et al., 2013), the major part of this delay must be due to engaged polymerases that continue to elongate after the parS-Homie-lacZ becomes unlinked from the eve enhancers. These results fit with a model in which sustained physical association of eve enhancers and the lac $Z$ promoter is necessary for continuous initiation of transcription.

\section{Physical enhancer-promoter engagement leads to distinct topological conformations}

While we have demonstrated that enhancer action depends upon sustained physical proximity to the promoter of the lac $Z$ reporter, the proximity generated by homie pairing alone is not sufficient. Among the 307 parS-homie-lacZ nuclei in which homie-pairing likely occurred (i.e. nuclei occupying the short-distance peak in the histogram in Fig. $3 \mathrm{~B}$ ), only $63 \%$ express lacZ-PP7 during the 30 min window of observation in n.c. 14. Notably, in the nuclei that show reporter activity the MS2-parS distance is $27 \pm 8 \mathrm{~nm}$ (mean \pm SEM) lower than the distance in the nuclei where the lac $Z$ reporter is inactive, suggesting that an additional compaction is associated with reporter activation.

The insufficiency of close proximity is even clearer when we employ the parS-homieR-lacZ reporter. This reporter still facilitates homie-pairing, yet likely renders the lac $Z$ and the eve enhancers on opposite sides of these paired boundary elements (Fig. 3D, Fujioka et al., 2016). Of the 54 nuclei in which the lac $Z$ transgene is close to the eve locus, there are only three in which the eve enhancers activate reporter transcription, and only for brief periods of $3-5$ min.

Is transcriptional activation associated with an additional step that promotes physical enhancer-promoter en-
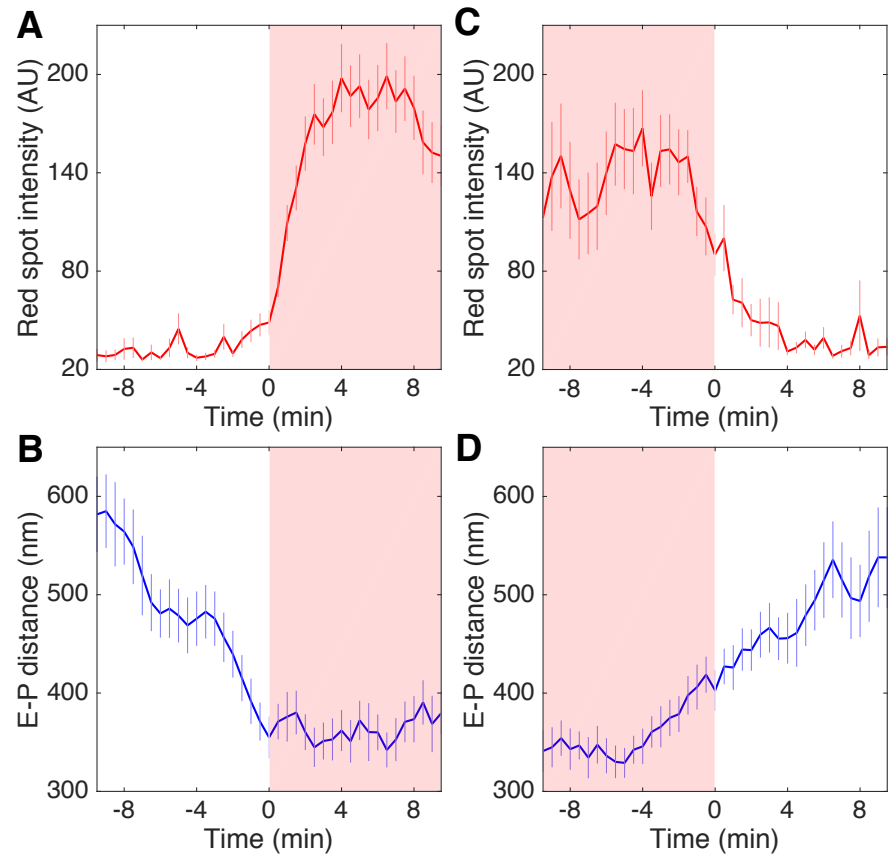

FIG. 4: Dynamics of chromatin movement underlies kinetics of enhancer-promoter interactions and transcriptional activation. (A) Average $l a c Z$ activity as a function of time for nuclei turning on (time traces for individual nuclei are aligned such that activity starts at 0 min, i.e. first occurrence of red signal). (B) RMS distance between blue (MS2) and green (parS) DNA foci (E-P distance) in nuclei corresponding to data in (A). (C) Average lacZ activity for nuclei turning off, aligned such that activity ends at 0 min. (D) RMS distance between blue (MS2) and green (parS) DNA foci in nuclei corresponding to data in $(\mathrm{C})$. In all panels shaded background signifies presence of $l a c Z$ activity.

gagement? We have shown that homie pairing establishes a new topological conformation in which the parS-homie-lacZ transgene and the eve locus are in close proximity (Fig. 3B). However, because the eve enhancers are distributed within 20kb of the eve-MS2 locus (Fig. 5A), homie pairing does not necessarily yield a close proximity between eve enhancers and the $l a c Z$ reporter. To further examine this point we take advantage of the property that independent eve enhancers regulate individual stripes of the eve pattern along the embryo (Fig. 5A). Thus by examining nuclei from different stripes separately we are able to explore the topology of the locus under different activating enhancers.

We first considered the distance of the eve-MS2 gene relative to the parS tag (blue-green) in nuclei where homie pairing occurs but lac $Z$ transcription is lacking (Red-OFF, Fig. 5B). We observed different distances in nuclei belonging to different stripes, i.e. a dependence of the spatial arrangement on the identity of the activating enhancers (Fig. 5C, Red-OFF). If an activating enhancer is physically engaged with the endogenous eve promoter, it colocalizes with the blue eve-MS2 focus. Thus the distance to the homie pair (green parS tag) should depend on the distance between the activating enhancer and the endogenous homie. Indeed, the distance between the eve gene and the parS tag of the inactive lacZ reporter in stripe 5 is 


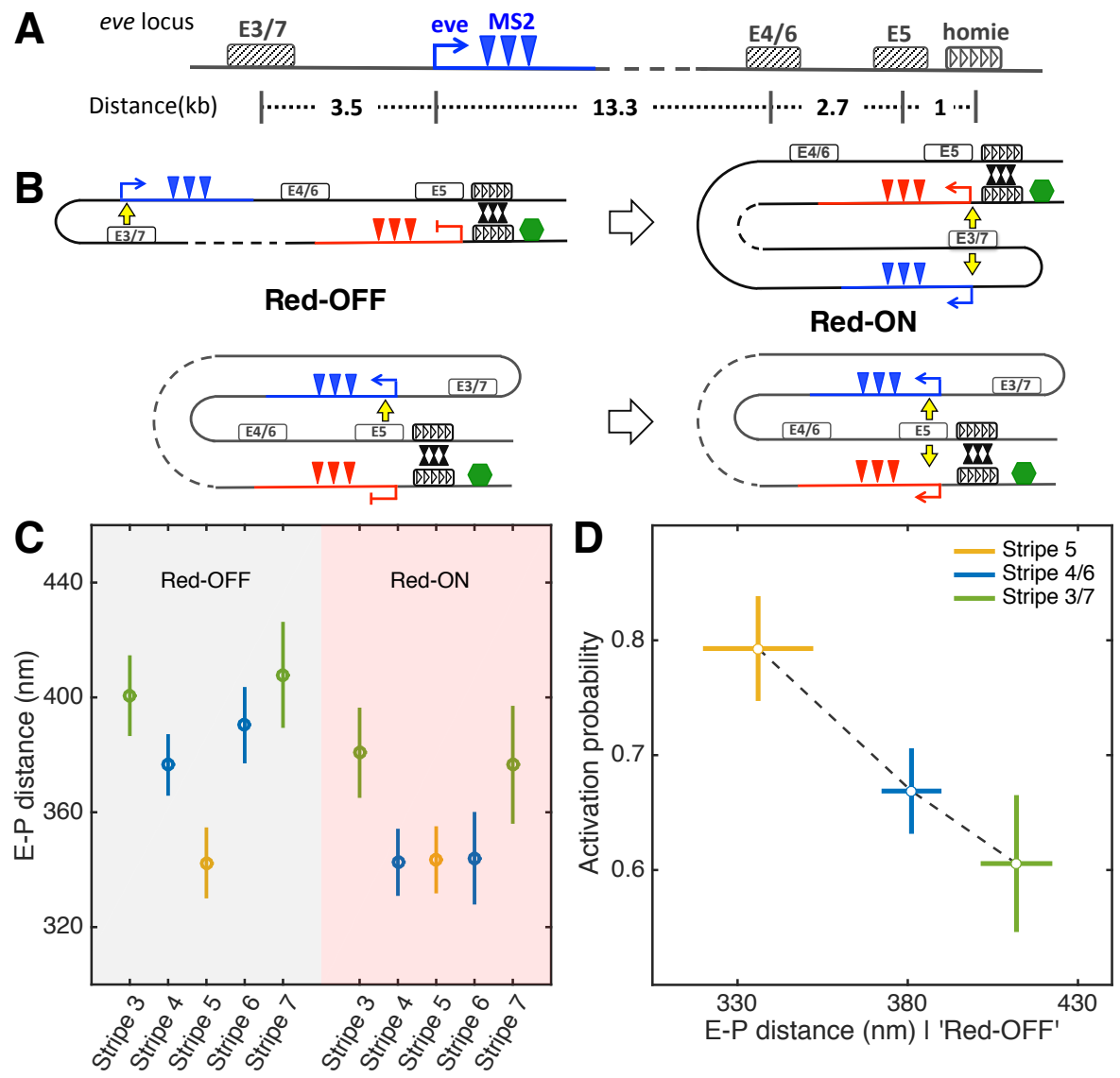

FIG. 5: Activation from endogenous enhancers is governed by enhancer-promoter distances. (A) Endogenous eve locus with stripe- $3 / 7$ enhancer, stripe- $4 / 6$ enhancer and stripe- 5 enhancer and their relative locations with respect to the eve promoter and the endogenous homie sequence. The stripe 5 and stripe $4 / 6$ enhancers are located downstream of the eve promoter; $1.0 \mathrm{~kb}$ and $3.7 \mathrm{~kb}$ upstream of, homie, respectively. The stripe $3 / 7$ enhancer is located upstream of the eve promoter; $20.5 \mathrm{~kb}$ from homie. (B) Cartoon showing eve locus under different looping conformations (Red-OFF and Red-ON) after homie-homie pairing. Top row is for stripe 3/7; bottom row is for stripe 5. (C) RMS distance between blue (MS2) and green (parS) DNA foci (E-P distance) for all nuclei in which homie-homie pairing occurs. RMS distances are calculated for individual eve stripes. Red-OFF and Red-ON correspond to the eve locus conformations illustrated in (B) for the parS-homie-lacZ transgene. (D) Fraction of homie-linked nuclei in each stripe that express lacZ (activation probability) is plotted as a function of distance as measured in (C). See also Fig. S4.

shorter than that observed for nuclei in stripes $4 / 6$ and $3 / 7$, for which the enhancers are located farther away from the parS tag (Fig. 5C). Moreover, for the two enhancers that drive eve expression in a pair of stripes, $4 / 6$ and $3 / 7$, the distance between eve and the parS tag is closely matched within each stripe pair. Finally, for embryos with the parShomieR-lacZ transgene the same trend is observed in nuclei with homie pairing (Fig. S4A). Taken together, these results argue strongly that the eve enhancers directly engage the endogenous eve promoter to activate transcription and that in each eve stripe a distinct topological conformation is adopted.

Next, if transcriptional activation entails physical engagement, in each stripe we expect that in nuclei where lac $Z$ transcription is active (Red-ON, Fig. 5B) not only will the eve-MS2 gene be in close proximity to the activating enhancer but also the parS-homie-lacZ reporter. Indeed, in the nuclei with the active reporter a significant shortening of the distance between the eve enhancers and the lac $Z$ promoter is observed in stripes $4 / 6$ and $3 / 7$ when lac $Z$ is activated (Fig. 5C), which further argues for transcription associated compaction of the locus. Critically, there is no lac $Z$ activity dependent shift apparent for stripe 5 nuclei as in these nuclei the eve stripe 5 enhancer is already in close proximity to the $l a c Z$ promoter.

Finally, a stripe-specific topology is also favored by observations in measurements of the activation probability of the promoter driving lac $Z$ expression. If transcription of the homie-linked reporters is linked to enhancer engagement, a plausible expectation is that activation frequency would be distance dependent. To assess the effect of distance we determined the fraction of homie-linked nuclei in each stripe that express lacZ. As predicted, the fraction of transcriptionally active reporters decreases with increasing distance between the stripe enhancer and the lacZ promoter (Fig. 5D). Stripe 5 has the highest activation probability ( $80 \%$ ), while stripes $3 / 7$ have the lowest probability of enhancer engagement with the lac $Z$ reporter. The fact that we observe a linearly decaying relationship is consistent with contact probability measurements (Lieberman-Aiden et al., 2009). Together, these results provide compelling evidence that transcriptional acti- 
vation entails physical enhancer-promoter engagement and is thereby associated with stripe-specific topological compaction of the eve locus.

\section{Promoter competition has phenotypic consequences}

In our experiments, the eve stripe enhancer drives expression from two different eve promoters, one for the endogenous eve gene and the other for the lac $Z$ reporter (Fig. $5 \mathrm{~B}$, Red-ON). If the activity of the stripe enhancers is limiting, the lac $Z$ reporter will reduce transcription of the eve gene. To determine whether promoter competition occurs in our genomic setup we compared eve transcription (i.e. the intensity of the blue MS2 signal) in individual nuclei in which $l a c Z$ is active and nuclei in which lacZ is silent (Fig. $6 \mathrm{~A}$, see Methods). For each eve stripe, we measured a 5\%$25 \%$ reduction in endogenous eve transcription in nuclei in which lac $Z$ is also transcribed compared to nuclei in which lac $Z$ is not transcribed. The average reduction per nucleus is highest for stripe 5, and lowest for stripes 3 and 7 .

Eve is a primary pair-rule gene that is responsible for segment patterning. To determine whether the observed reduction in eve transcription has any phenotypic consequences, we crossed males carrying a tagless homie-lac $Z$ transgene at $-142 \mathrm{~kb}$ (Fig. 1A) to females heterozygous for a wild type eve gene and an eve deficiency $(D f(2 R)$ eve $)$. eve is weakly haploinsufficient and $7 \%$ of $+/ D f(2 R)$ eve flies display patterning defects in even-numbered abdominal segments. Consistent with the reduction in the level of eve nascent transcripts, the presence of the homie-lacZ transgene exacerbates eve haploinsufficiency (Fig. 6B-D). Altogether $29 \%$ of the Homie-lacZ/Df(2R)eve flies have abdominal defects which corresponds to a 4 -fold increase (Fig. $6 \mathrm{E}$ ). Taken together, the above results suggest that competition between two promoters at the transcriptional level in the early embryo has phenotypic consequences for patterning in the adult. These findings provide evidence that manipulating topological chromatin structures can interfere with developmental programs.

\section{DISCUSSION}

Despite extensive studies over more than three decades, many questions still remain regarding how enhancers communicate with their target promoters over large genomic distances. Critically, although recent FISH and 3C-based genomic experiments have provided extensive evidence supporting physical interactions for long-range enhancerpromoter communication (Deng et al., 2012; Fabre et al., 2015; Hnisz et al., 2016; Ji et al., 2016; Phillips-Cremins et al., 2013; Rao et al., 2014; Williamson et al., 2016), we still miss a dynamic picture that could disentangle cause from consequence and that could distinguish transient contact with formation of stable topological structures. Here, we have developed a live imaging approach on a well-studied endogenous locus that allows us to probe the dynamics of topological chromatin structures at the single-cell level and its impact on transcriptional activity. We show directly that sustained physical proximity is necessary for enhancer
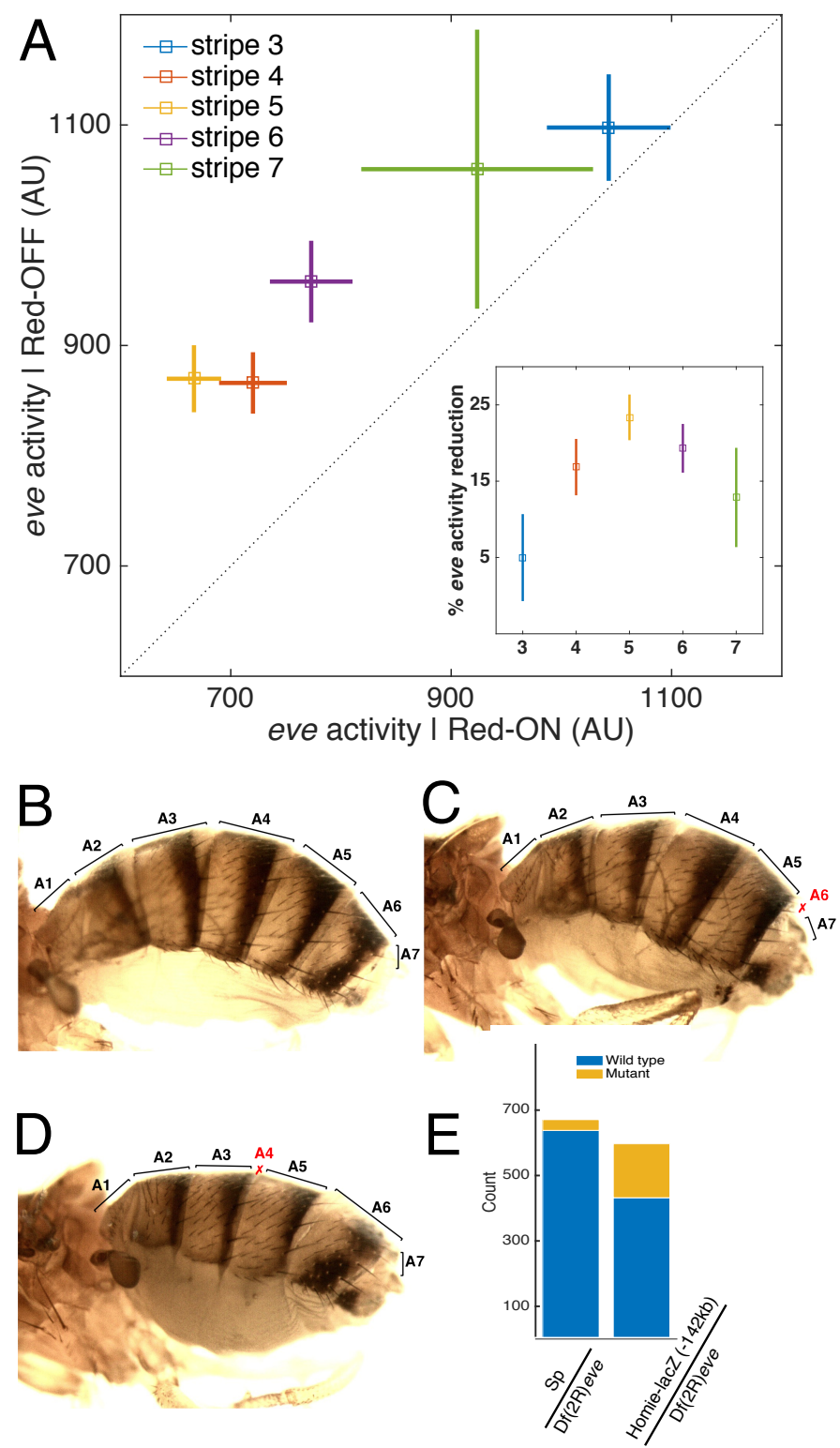

FIG. 6: Long-distance-mediated promoter competition results in patterning phenotypes. (A) Endogenous eve activity in nuclei that also display lac $Z$ activity (x-axis) is lower than in nuclei where $l a c Z$ is not expressed (y-axis). Inset: Reduction in eve activity for each stripe. (B-D) Adult wild-type (B) and mutant (C and D) flies from crosses between $\mathrm{Sp} /$ Homie-lacZ males and $\mathrm{CyO} / \mathrm{Df}(2 \mathrm{R})$ eve females. (C) and (D) show defects in abdominal segments A4 and A6, respectively, resulting from reduced eve activity in stripe 5 and stripe 6 , respectively. Abdominal segments are labeled, with defective segments marked in red. (E) Results of phenotype scoring. Mutant counts include both A4 and A6 phenotype. p value from Fishers exact test.

action, and distinct topologies are observed for different pairing of the promoter with an activating enhancer, even when enhancers are clustered together. Though all our results are based on a specific experimental system it seems likely that they will be more broadly applicable.

To examine long-range transcriptional activation we placed a reporter gene at $150 \mathrm{~kb}$ distance of the presumptive eve enhancers. Although such distance is un- 
usually large for enhancer-promoter interactions in flies, in higher eukaryotes enhancers are known to function over comparable or greater distances (Sanyal et al., 2012). At such distance the chromatin fiber can display fast random movements, which creates an entropic difficulty for specific long-range chromatin interactions and thus a kinetic barrier for the establishment of a productive pre-initiation complex. Architectural proteins have been suggested to increase contact frequency between chromatin loci separated by large genomic distances and facilitated the formation of sustained physical proximity permitting functional enhancer-promoter interactions (Erokhin et al., 2011; Shen et al., 2012; Symmons et al., 2014). The subdivision of genomes to topologically associating domains (TADs) was further suggested to facilitate local (intra-domain) chromatin interactions and reduce the search space, as exemplified for VDJ recombination in the mammalian immune system (Lucas et al., 2014).

To facilitate the interaction between the enhancers and the reporter in our experimental setup, we thus employ the homie element in our transgene, thereby inducing a stable loop conformation with the endogenous homie element that demarcates the 3 end of the eve locus. This element was shown to be bound by a wide array of Drosophila insulator proteins in the blastoderm embryo, including $\mathrm{Su}(\mathrm{Hw})$, CP190, BEAF-32 and dCTCF (Negre et al., 2010). homiehomie pairing between the transgene and the eve locus provides spatial proximity that we find to be necessary to allow the enhancers to activate transcription of the reporter. When the homie element in the transgene is replaced by lambda DNA, transcriptional activity of the reporter gene is not observed. As this transgene should encounter the eve enhancers with roughly the same frequency as the transgenes carrying homie, it demonstrates that transient encounters are not sufficient to activate PolII transcription, and the various transcription factors associated with the eve enhancers and the eve promoter are unable to establish stable and/or productive enhancer-promoter contacts on their own.

Another example in which an architectural element is deployed de novo to stabilize long distance enhancerpromoter interactions is the variably occupied tissue specific dCTCF binding site in the Ultrabithorax intron just upstream of the abx/bx enhancer (Magbanua et al., 2015). More generally, TAD boundaries in mammalian genomes often harbor CTCF binding sites, which are believed to play a major role in genome organization. Intriguingly, these sites were recently shown to have a specific orientation (Guo et al., 2015; Rao et al., 2014). When we examine a reporter construct in which we inverted homie orientation we find that while pairing is still maintained, the frequency of transcriptional activation drops significantly. The cause is likely because the enhancers and the promoters are now found on opposite sides of the paired insulators (Fujioka et al., 2016). Thus, alteration to these binding sites can bare important functional implications and indeed many cancer types accumulate CTCF binding site mutations that modulate chromatin conformations with subsequent influences on transcription (Flavahan et al., 2016; Katainen et al., 2015). Our experimental setup provides an opportunity for exploring the dynamic relationship between topo- logical alterations and transcriptional regulation resulting from mutations in boundary elements.

Overall, our experiments demonstrate that physical proximity is a necessary condition for the eve enhancers to activate lac $Z$ expression. Importantly, we find that transcription ceases upon dissociation of the enhancers and the reporter, suggesting a requirement for sustained physical proximity, and excluding mechanisms of transient enhancer promoter associations leading to stable activity. While we observe this tight relationship between physical proximity and activation, for other genes, loop formation could be a permissive step, with pre-formed enhancerpromoter interactions preceding transcriptional activation (de Laat and Duboule, 2013; Ghavi-Helm et al., 2014; Jin et al., 2013; Montavon et al., 2011). This discrepancy might reflect regulatory strategies that are gene or developmental context dependent. Nevertheless, consistent with the observations that a large amount of reorganization in enhancer-promoter interactions exist upon tissue differentiation (Dixon et al., 2015; Javierre et al., 2016), our results show that establishment of physical interactions between enhancers and promoters could be a rate-limiting step for transcriptional activation and play a key role in regulating transcription and cell fate.

Applying our single-cell live imaging approach in the early fly embryo offers an unprecedented opportunity to examine topological chromatin structures of a locus in a cell-specific way. We provide compelling evidence that eve expressing cells adopt distinct topological chromatin structures according to the stripe-specific identity of the activating enhancer. Notably the eve enhancers are located within $10 \mathrm{~kb}$ of the endogenous eve promoter and the homie-paired promoter of the reporter gene. A possible model could be that the adjacent eve enhancers will be found in close proximity to the promoters within a local environment permitting any of the enhancers to activate either of the promoters without further topological distinctions. Our observation of a stripe-specific distance between the locus of the eve gene and the homie-tethered parS tag, and the further compaction of the locus upon concurrent activation of the reporter, seem to exclude such a model. But rather this seems to suggest the necessity of a specific enhancer-promoter engagement for transcriptional activation, even within a relatively short genomic range.

What is the spatial scale that defines enhancer-promoter engagement? Transcriptional activity in our experimental system is only observed when the reporter moves into close proximity to the eve locus. The transition from inactivate to active occurs when the parS tag comes within $350 \mathrm{~nm}$ of the eve gene. When the reporter moves more than 350 $\mathrm{nm}$ away from the eve gene, transcription shuts off, and already engaged Pol II bring their elongation process to completion. The range of the observed necessary physical proximity is likely to be dictated by the spatial scale set up by the size of the protein complexes that are believed to physically bridge enhancers with promoters (¿2MDa with diameter ¿20nm (Dill et al., 2011)), for example Mediator complex and the Pol II pre-initiation complex (Kagey et al., 2010; Plaschka et al., 2015; Robinson et al., 2016). This requirement of $350 \mathrm{~nm}$ in enhancer-promoter proximity in order to activate transcription is comparable with DNA 
FISH measurements in mammalian systems that estimate the proximity between the shh promoter and its enhancers in mouse limbs (Amano et al., 2009); (Williamson et al., 2016).

Finally, when the endogenous eve locus is presented with an extra ectopic promoter, its activation results in concomitant reduction in endogenous eve transcription, phenotypically leading to patterning defects in the corresponding segments. Usually, competing promoters are physically linked within short genomic distances ( $\mathrm{i} 20 \mathrm{~kb})$ (Foley and Engel, 1992; Fukaya et al., 2016). However, our results show that physically associating the competing promoters could in principle also be achieved through dynamic alterations of chromatin conformations. This observation reinforces the notion that rearrangement of topological chromatin structures is able to re-wire enhancer-promoter interactions, and that the rewiring can cause new phenotypes in many developmental contexts. For example in humans, structural chromosome variants that disrupt boundary elements flanking TADs may lead to diseases (Franke et al., 2016; Lupianez et al., 2015). Thus our experiments provide a potential path to synthetically rewire enhancer-promoter interactions to perturb chromatin topologies and thereby interfere with developmental programs (Deng et al., 2012; Deng et al., 2014),

In conclusion, we propose a methodological framework for investigating the relationship between chromatin dynamics and long-range enhancer-mediated transcriptional regulation. With the ability of tracing endogenous loci in individual cells and simultaneously measuring temporal dynamics and spatial information, new mechanistic insights into enhancer-promoter interactions are likely to be uncovered.

\section{Methods Summary}

\author{
Plasmid construction \\ Transgenic fly generation \\ Fluorescent in-situ hybridization \\ Phenotypic scoring \\ Microscopy and imaging conditions \\ Image processing and data analysis
}

\section{Acknowledgements}

We thank P. Schedl for bringing the eve-homie system to our attention. We thank K. Bystricky for introducing us to the ParB/ParS system, and F. Payre and P. Valenti for sharing a ParB-eGFP plasmid and the parS sequence. We thank P. Ratchasanmuang and S. Ryabichko for assistance with cloning and fly husbandry, and the Bloomington Drosophila Stock Center for fly strains. We are also grateful to L. Barinov, S. Blythe, M. Levine, M. Levo, P. Schedl, E.F. Wieschaus for discussion and help with the manuscript. This study was funded by grants from the National Institutes of Health (U01 EB021239, R01 GM097275, and R01 GM050231). HC was supported by the Charles H. Revson Biomedical Science Fellowship.

\section{References}

Amano, T., Sagai, T., Tanabe, H., Mizushina, Y., Nakazawa, H., and Shiroishi, T. (2009). Chromosomal dynamics at the Shh locus: limb bud-specific differential regulation of competence and active transcription. Dev Cell $16,47-57$.

Arnold, C.D., Gerlach, D., Stelzer, C., Boryn, L.M., Rath, M., and Stark, A. (2013). Genome-wide quantitative enhancer activity maps identified by STARR-seq. Science 339, 1074-1077.

Bateman, J.R., Lee, A.M., and Wu, C.T. (2006). Sitespecific transformation of Drosophila via phiC31 integrasemediated cassette exchange. Genetics 173, 769-777.

Benabdallah, N.S., and Bickmore, W.A. (2015). Regulatory Domains and Their Mechanisms. Cold Spring Harb Symp Quant Biol 80, 45-51. Benoist, C., and Chambon, P. (1981). In vivo sequence requirements of the SV40 early promotor region. Nature 290, 304-310.

Bertrand, E., Chartrand, P., Schaefer, M., Shenoy, S.M., Singer, R.H., and Long, R.M. (1998). Localization of ASH1 mRNA particles in living yeast. Mol Cell 2, 437-445.

Blackwood, E.M., and Kadonaga, J.T. (1998). Going the distance: a current view of enhancer action. Science 281, 60-63. Buecker, C., and Wysocka, J. (2012). Enhancers as information integration hubs in development: lessons from genomics. Trends Genet 28, 276-284.

Bystricky, K. (2015). Chromosome dynamics and folding in eukaryotes: Insights from live cell microscopy. FEBS Lett 589, 3014-3022.

Consortium, E.P. (2012). An integrated encyclopedia of DNA elements in the human genome. Nature 489, 57-74.

de Laat, W., and Duboule, D. (2013). Topology of mammalian developmental enhancers and their regulatory landscapes. Nature 502, 499-506.

Deng, W., Lee, J., Wang, H., Miller, J., Reik, A., Gregory, P.D., Dean, A., and Blobel, G.A. (2012). Controlling long-range genomic interactions at a native locus by targeted tethering of a looping factor. Cell 149, 1233-1244.

Deng, W., Rupon, J.W., Krivega, I., Breda, L., Motta, I., Jahn, K.S., Reik, A., Gregory, P.D., Rivella, S., Dean, A., et al. (2014). Reactivation of developmentally silenced globin genes by forced chromatin looping. Cell 158, 849860.

Dill, K.A., Ghosh, K., and Schmit, J.D. (2011). Physical limits of cells and proteomes. Proc Natl Acad Sci U S A 108, 17876-17882.

Dixon, J.R., Jung, I., Selvaraj, S., Shen, Y., AntosiewiczBourget, J.E., Lee, A.Y., Ye, Z., Kim, A., Rajagopal, N., Xie, W., et al. (2015). Chromatin architecture reorganization during stem cell differentiation. Nature 518, 331-336.

Dubarry, M., Loiodice, I., Chen, C.L., Thermes, C., and Taddei, A. (2011). Tight protein-DNA interactions favor gene silencing. Genes Dev 25, 1365-1370.

Dubarry, N., Pasta, F., and Lane, D. (2006). ParABS systems of the four replicons of Burkholderia cenocepacia: new chromosome centromeres confer partition specificity. J Bacteriol 188, 1489-1496.

Dubuis, J.O., Samanta, R., and Gregor, T. (2013). Accurate measurements of dynamics and reproducibility in small genetic networks. Mol Syst Biol 9, 639. 
Erokhin, M., Davydova, A., Kyrchanova, O., Parshikov, A., Georgiev, P., and Chetverina, D. (2011). Insulators form gene loops by interacting with promoters in Drosophila. Development 138, 4097-4106.

Fabre, P.J., Benke, A., Joye, E., Nguyen Huynh, T.H., Manley, S., and Duboule, D. (2015). Nanoscale spatial organization of the HoxD gene cluster in distinct transcriptional states. Proc Natl Acad Sci U S A 112, 13964-13969.

Flavahan, W.A., Drier, Y., Liau, B.B., Gillespie, S.M., Venteicher, A.S., Stemmer-Rachamimov, A.O., Suva, M.L., and Bernstein, B.E. (2016). Insulator dysfunction and oncogene activation in IDH mutant gliomas. Nature 529, 110-114.

Foley, K.P., and Engel, J.D. (1992). Individual stage selector element mutations lead to reciprocal changes in beta- vs. epsilon-globin gene transcription: genetic confirmation of promoter competition during globin gene switching. Genes Dev 6, 730-744.

Franke, M., Ibrahim, D.M., Andrey, G., Schwarzer, W., Heinrich, V., Schopflin, R., Kraft, K., Kempfer, R., Jerkovic, I., Chan, W.L., et al. (2016). Formation of new chromatin domains determines pathogenicity of genomic duplications. Nature 538, 265-269.

Fujioka, M., Mistry, H., Schedl, P., and Jaynes, J.B. (2016). Determinants of Chromosome Architecture: Insulator Pairing in cis and in trans. PLoS Genet 12, e1005889.

Fujioka, M., Sun, G., and Jaynes, J.B. (2013). The Drosophila eve insulator Homie promotes eve expression and protects the adjacent gene from repression by polycomb spreading. PLoS Genet 9, e1003883.

Fujioka, M., Wu, X., and Jaynes, J.B. (2009). A chromatin insulator mediates transgene homing and very longrange enhancer-promoter communication. Development 136, 3077-3087.

Fukaya, T., Lim, B., and Levine, M. (2016). Enhancer Control of Transcriptional Bursting. Cell 166, 358-368.

Gao, Y., and Kilfoil, M.L. (2009). Accurate detection and complete tracking of large populations of features in three dimensions. Opt Express 17, 4685-4704.

Garcia, H.G., Tikhonov, M., Lin, A., and Gregor, T. (2013). Quantitative imaging of transcription in living Drosophila embryos links polymerase activity to patterning. Curr Biol 23, 2140-2145.

Gasser, S.M. (2002). Visualizing chromatin dynamics in interphase nuclei. Science 296, 1412-1416.

Ghavi-Helm, Y., Klein, F.A., Pakozdi, T., Ciglar, L., Noordermeer, D., Huber, W., and Furlong, E.E. (2014). Enhancer loops appear stable during development and are associated with paused polymerase. Nature 512, 96-100.

Guo, Y., Xu, Q., Canzio, D., Shou, J., Li, J., Gorkin, D.U., Jung, I., Wu, H., Zhai, Y., Tang, Y., et al. (2015). CRISPR Inversion of CTCF Sites Alters Genome Topology and Enhancer/Promoter Function. Cell 162, 900-910.

Hnisz, D., Day, D.S., and Young, R.A. (2016). Insulated Neighborhoods: Structural and Functional Units of Mammalian Gene Control. Cell 167, 1188-1200.

Hocine, S., Raymond, P., Zenklusen, D., Chao, J.A., and Singer, R.H. (2013). Single-molecule analysis of gene expression using two-color RNA labeling in live yeast. Nat Methods 10, 119-121.

Javierre, B.M., Burren, O.S., Wilder, S.P., Kreuzhuber,
R., Hill, S.M., Sewitz, S., Cairns, J., Wingett, S.W., Varnai, C., Thiecke, M.J., et al. (2016). Lineage-Specific Genome Architecture Links Enhancers and Non-coding Disease Variants to Target Gene Promoters. Cell 167, 1369$1384 \mathrm{e} 1319$.

Ji, X., Dadon, D.B., Powell, B.E., Fan, Z.P., BorgesRivera, D., Shachar, S., Weintraub, A.S., Hnisz, D., Pegoraro, G., Lee, T.I., et al. (2016). 3D Chromosome Regulatory Landscape of Human Pluripotent Cells. Cell Stem Cell 18, 262-275.

Jin, F., Li, Y., Dixon, J.R., Selvaraj, S., Ye, Z., Lee, A.Y., Yen, C.A., Schmitt, A.D., Espinoza, C.A., and Ren, B. (2013). A high-resolution map of the three-dimensional chromatin interactome in human cells. Nature 503, 290294.

Kagey, M.H., Newman, J.J., Bilodeau, S., Zhan, Y., Orlando, D.A., van Berkum, N.L., Ebmeier, C.C., Goossens, J., Rahl, P.B., Levine, S.S., et al. (2010). Mediator and cohesin connect gene expression and chromatin architecture. Nature 467, 430-435.

Katainen, R., Dave, K., Pitkanen, E., Palin, K., Kivioja, T., Valimaki, N., Gylfe, A.E., Ristolainen, H., Hanninen, U.A., Cajuso, T., et al. (2015). CTCF/cohesin-binding sites are frequently mutated in cancer. Nat Genet 47,818 821.

Kvon, E.Z., Kazmar, T., Stampfel, G., Yanez-Cuna, J.O., Pagani, M., Schernhuber, K., Dickson, B.J., and Stark, A. (2014). Genome-scale functional characterization of Drosophila developmental enhancers in vivo. Nature 512, 91-95.

Larson, D.R., Zenklusen, D., Wu, B., Chao, J.A., and Singer, R.H. (2011). Real-time observation of transcription initiation and elongation on an endogenous yeast gene. Science 332, 475-478.

Lee, A.M., and Wu, C.T. (2006). Enhancerpromoter communication at the yellow gene of Drosophila melanogaster: diverse promoters participate in and regulate trans interactions. Genetics 174, 1867-1880.

Lettice, L.A., Heaney, S.J., Purdie, L.A., Li, L., de Beer, P., Oostra, B.A., Goode, D., Elgar, G., Hill, R.E., and de Graaff, E. (2003). A long-range Shh enhancer regulates expression in the developing limb and fin and is associated with preaxial polydactyly. Hum Mol Genet 12, 1725-1735.

Levine, M. (2010). Transcriptional enhancers in animal development and evolution. Curr Biol 20, R754-763.

Levine, M., Cattoglio, C., and Tjian, R. (2014). Looping back to leap forward: transcription enters a new era. Cell 157, 13-25.

Lewis, E.B. (1978). A gene complex controlling segmentation in Drosophila. Nature 276, 565-570.

Lieberman-Aiden, E., van Berkum, N.L., Williams, L., Imakaev, M., Ragoczy, T., Telling, A., Amit, I., Lajoie, B.R., Sabo, P.J., Dorschner, M.O., et al. (2009). Comprehensive mapping of long-range interactions reveals folding principles of the human genome. Science 326, 289-293.

Little, S.C., Tikhonov, M., and Gregor, T. (2013). Precise developmental gene expression arises from globally stochastic transcriptional activity. Cell 154, 789-800.

Little, S.C., Tkacik, G., Kneeland, T.B., Wieschaus, E.F., and Gregor, T. (2011). The formation of the Bicoid morphogen gradient requires protein movement from 
anteriorly localized mRNA. PLoS Biol 9, e1000596.

Long, H.K., Prescott, S.L., and Wysocka, J. (2016). Ever-Changing Landscapes: Transcriptional Enhancers in Development and Evolution. Cell 167, 1170-1187.

Lucas, J.S., Zhang, Y., Dudko, O.K., and Murre, C. (2014). 3D trajectories adopted by coding and regulatory DNA elements: first-passage times for genomic interactions. Cell 158, 339-352.

Lupianez, D.G., Kraft, K., Heinrich, V., Krawitz, P., Brancati, F., Klopocki, E., Horn, D., Kayserili, H., Opitz, J.M., Laxova, R., et al. (2015). Disruptions of topological chromatin domains cause pathogenic rewiring of geneenhancer interactions. Cell 161, 1012-1025.

Magbanua, J.P., Runneburger, E., Russell, S., and White, R. (2015). A variably occupied CTCF binding site in the ultrabithorax gene in the Drosophila bithorax complex. Mol Cell Biol 35, 318-330.

Markenscoff-Papadimitriou, E., Allen, W.E., Colquitt, B.M., Goh, T., Murphy, K.K., Monahan, K., Mosley, C.P., Ahituv, N., and Lomvardas, S. (2014). Enhancer interaction networks as a means for singular olfactory receptor expression. Cell 159, 543-557.

Mifsud, B., Tavares-Cadete, F., Young, A.N., Sugar, R., Schoenfelder, S., Ferreira, L., Wingett, S.W., Andrews, S., Grey, W., Ewels, P.A., et al. (2015). Mapping long-range promoter contacts in human cells with high-resolution capture Hi-C. Nat Genet 47, 598-606.

Montavon, T., Soshnikova, N., Mascrez, B., Joye, E., Thevenet, L., Splinter, E., de Laat, W., Spitz, F., and Duboule, D. (2011). A regulatory archipelago controls Hox genes transcription in digits. Cell 147, 1132-1145.

Negre, N., Brown, C.D., Shah, P.K., Kheradpour, P., Morrison, C.A., Henikoff, J.G., Feng, X., Ahmad, K., Russell, S., White, R.A., et al. (2010). A comprehensive map of insulator elements for the Drosophila genome. PLoS Genet 6, e1000814.

Phillips-Cremins, J.E., Sauria, M.E., Sanyal, A., Gerasimova, T.I., Lajoie, B.R., Bell, J.S., Ong, C.T., Hookway, T.A., Guo, C., Sun, Y., et al. (2013). Architectural protein subclasses shape 3D organization of genomes during lineage commitment. Cell 153, 1281-1295.

Plaschka, C., Lariviere, L., Wenzeck, L., Seizl, M., Hemann, M., Tegunov, D., Petrotchenko, E.V., Borchers, C.H., Baumeister, W., Herzog, F., et al. (2015). Architecture of the RNA polymerase II-Mediator core initiation complex. Nature 518, 376-380.

Qian, S., Capovilla, M., and Pirrotta, V. (1993). Molecular mechanisms of pattern formation by the BRE enhancer of the Ubx gene. EMBO J 12, 3865-3877.

Rao, S.S., Huntley, M.H., Durand, N.C., Stamenova, E.K., Bochkov, I.D., Robinson, J.T., Sanborn, A.L., Machol, I., Omer, A.D., Lander, E.S., et al. (2014). A 3D map of the human genome at kilobase resolution reveals principles of chromatin looping. Cell 159, 1665-1680.

Robinson, P.J., Trnka, M.J., Bushnell, D.A., Davis, R.E., Mattei, P.J., Burlingame, A.L., and Kornberg, R.D. (2016). Structure of a Complete Mediator-RNA Polymerase II PreInitiation Complex. Cell 166, 1411-1422 e1416.

Saad, H., Gallardo, F., Dalvai, M., Tanguy-le-Gac, N., Lane, D., and Bystricky, K. (2014). DNA dynamics dur- ing early double-strand break processing revealed by nonintrusive imaging of living cells. PLoS Genet 10, e1004187.

Sagai, T., Hosoya, M., Mizushina, Y., Tamura, M., and Shiroishi, T. (2005). Elimination of a long-range cisregulatory module causes complete loss of limb-specific Shh expression and truncation of the mouse limb. Development 132, 797-803.

Sanyal, A., Lajoie, B.R., Jain, G., and Dekker, J. (2012). The long-range interaction landscape of gene promoters. Nature 489, 109-113.

Shen, Y., Yue, F., McCleary, D.F., Ye, Z., Edsall, L., Kuan, S., Wagner, U., Dixon, J., Lee, L., Lobanenkov, V.V., et al. (2012). A map of the cis-regulatory sequences in the mouse genome. Nature 488, 116-120.

Sinclair, P., Bian, Q., Plutz, M., Heard, E., and Belmont, A.S. (2010). Dynamic plasticity of large-scale chromatin structure revealed by self-assembly of engineered chromosome regions. J Cell Biol 190, 761-776.

Sladitschek, H.L., and Neveu, P.A. (2015). MXSChaining: A Highly Efficient Cloning Platform for Imaging and Flow Cytometry Approaches in Mammalian Systems. PLoS One 10, e0124958.

Small, S. (2000). In vivo analysis of lacZ fusion genes in transgenic Drosophila melanogaster. Methods Enzymol 326, 146-159.

Spitz, F. (2016). Gene regulation at a distance: From remote enhancers to 3D regulatory ensembles. Semin Cell Dev Biol 57, 57-67.

Symmons, O., Uslu, V.V., Tsujimura, T., Ruf, S., Nassari, S., Schwarzer, W., Ettwiller, L., and Spitz, F. (2014). Functional and topological characteristics of mammalian regulatory domains. Genome Res 24, 390-400.

Tolhuis, B., Palstra, R.J., Splinter, E., Grosveld, F., and de Laat, W. (2002). Looping and interaction between hypersensitive sites in the active beta-globin locus. Mol Cell 10, 1453-1465.

Uslu, V.V., Petretich, M., Ruf, S., Langenfeld, K., Fonseca, N.A., Marioni, J.C., and Spitz, F. (2014). Long-range enhancers regulating Myc expression are required for normal facial morphogenesis. Nat Genet 46, 753-758.

Vodala, S., Abruzzi, K.C., and Rosbash, M. (2008). The nuclear exosome and adenylation regulate posttranscriptional tethering of yeast GAL genes to the nuclear periphery. Mol Cell 31, 104-113.

Williamson, I., Lettice, L.A., Hill, R.E., and Bickmore, W.A. (2016). Shh and ZRS enhancer colocalisation is specific to the zone of polarising activity. Development 143, 2994-3001.

Wu, B., Chen, J., and Singer, R.H. (2014). Background free imaging of single mRNAs in live cells using split fluorescent proteins. Sci Rep 4, 3615.

Yunger, S., Rosenfeld, L., Garini, Y., and Shav-Tal, Y. (2010). Single-allele analysis of transcription kinetics in living mammalian cells. Nat Methods 7, 631-633.

Zhang, Y., Wong, C.H., Birnbaum, R.Y., Li, G., Favaro, R., Ngan, C.Y., Lim, J., Tai, E., Poh, H.M., Wong, E., et al. (2013). Chromatin connectivity maps reveal dynamic promoter-enhancer long-range associations. Nature 504, 306-310. 
bioRxiv preprint doi: https://doi.org/10.1101/099523; this version posted January 31, 2017. The copyright holder for this preprint (which was not certified by peer review) is the author/funder, who has granted bioRxiv a license to display the preprint in perpetuity. It is made available under aCC-BY-NC 4.0 International license.

A

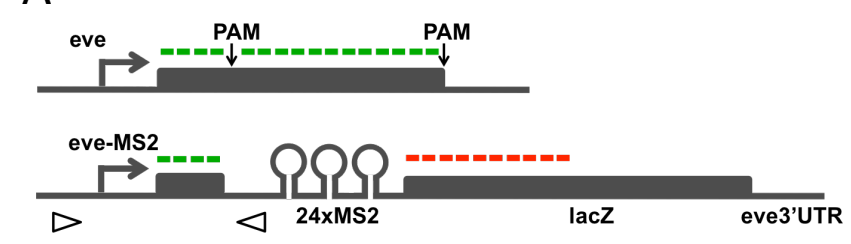

B
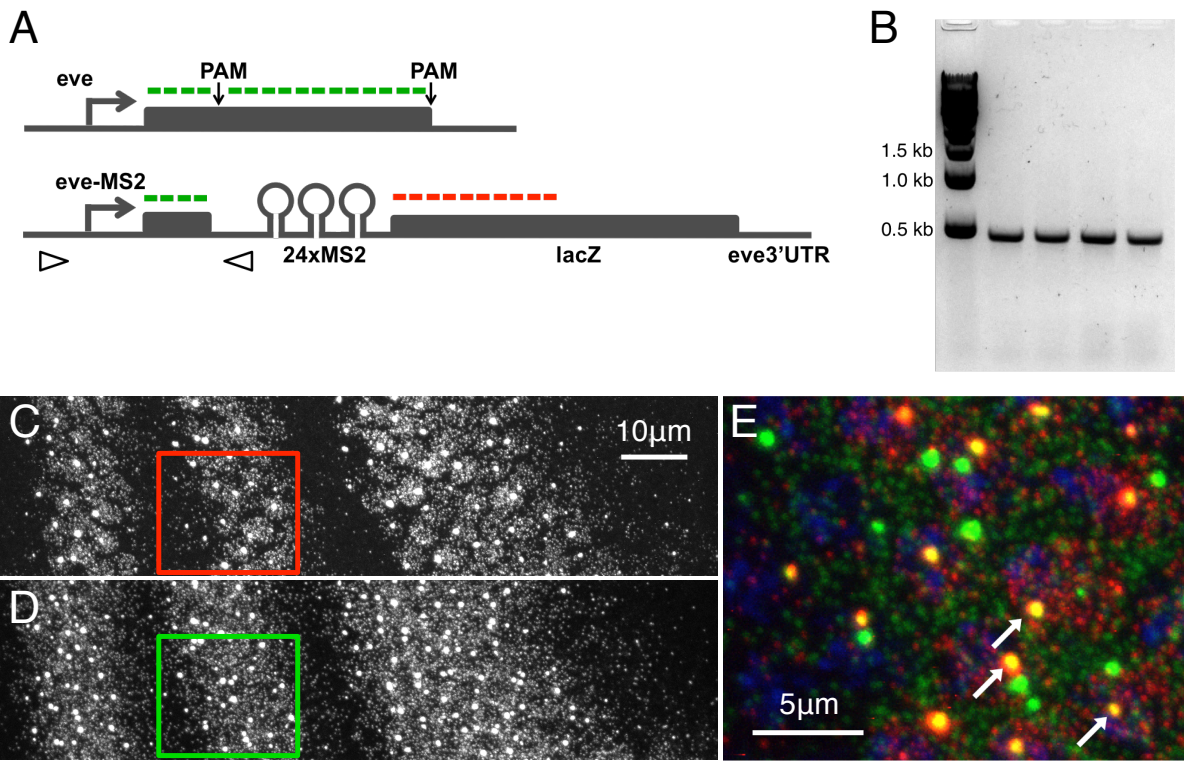

G

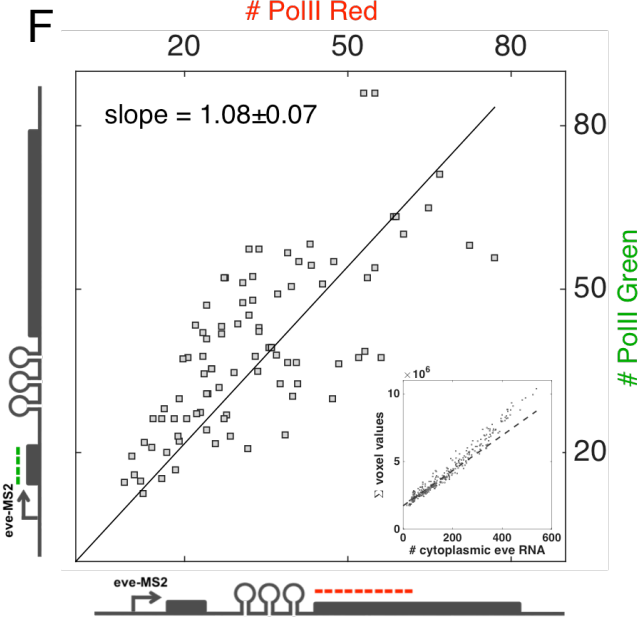

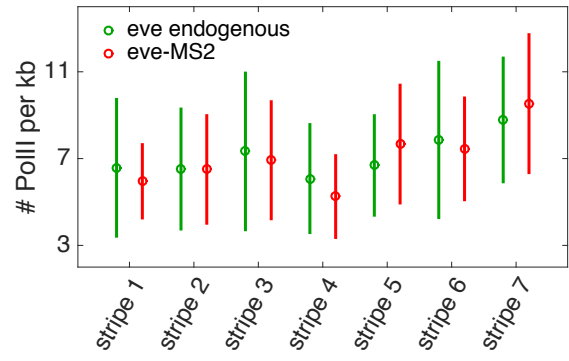

SUPP. FIG. 1: eve-MS2 allele recapitulates the expression pattern and transcriptional activity of the endogenous eve gene. (A) Editing the endogenous eve locus (upper) to obtain the eve-MS2 allele (bottom). Arrowheads indicate primers for PCR genotyping. Green and red lines mark sequences targeted by smFISH probes (eve-atto633 and lacZ-atto565, respectively). (B) Genotyping the eve-MS2 allele. PCR results from four independent transformant lines are shown. (C-G) FISH quantification of the transcriptional activity of the eve-MS2 allele. Maximum Z-projections are shown for lacZ-atto565 channel (C) and eve-atto633 channel (D) of an eve-MS2/eve+ embryo. Three eve stripes (stripe 5-7 from left to right) at 45 min in nuclear cycle 14 are shown. (E) Magnified view of square in C/D. Arrows indicate eve-MS2 transcription loci that are labeled by both probes. (F) Cytoplasmic spots and active transcription spots are identified by image analysis routines (see Methods). A cytoplasmic unit (CU) that corresponds to fluorescent intensity of a single cytoplasmic mRNA is extracted. Panel shows number of RNA polymerase II (Pol II) on the eve-MS2 loci as in inferred from either the CU derived from lacZ-atto565 (x-axis) or from eve-atto633 (y-axis) measurements. Inset shows calculation of cytoplasmic unit for eve. Specifically, a sliding window of 220x220x23 pixel (16.5x16.5x7.4 $\mu \mathrm{m}^{3}$ ) was applied to the raw image stack (C and D) and the total pixel values in the window were plotted against the number of cytoplasmic spots found in the window. A linear fit in the range of 0-100 cytoplasmic spots was applied to extract CU for each probe set. (G) Comparison of the PolII number on the eve-MS2 locus and on the endogenous eve locus. Note that the numbers reported in $(\mathrm{F})$ and $(\mathrm{G})$ are for two sister chromatids. 
bioRxiv preprint doi: https://doi.org/10.1101/099523; this version posted January 31, 2017. The copyright holder for this preprint (which was not certified by peer review) is the author/funder, who has granted bioRxiv a license to display the preprint in perpetuity. It is made available under aCC-BY-NC 4.0 International license.

A
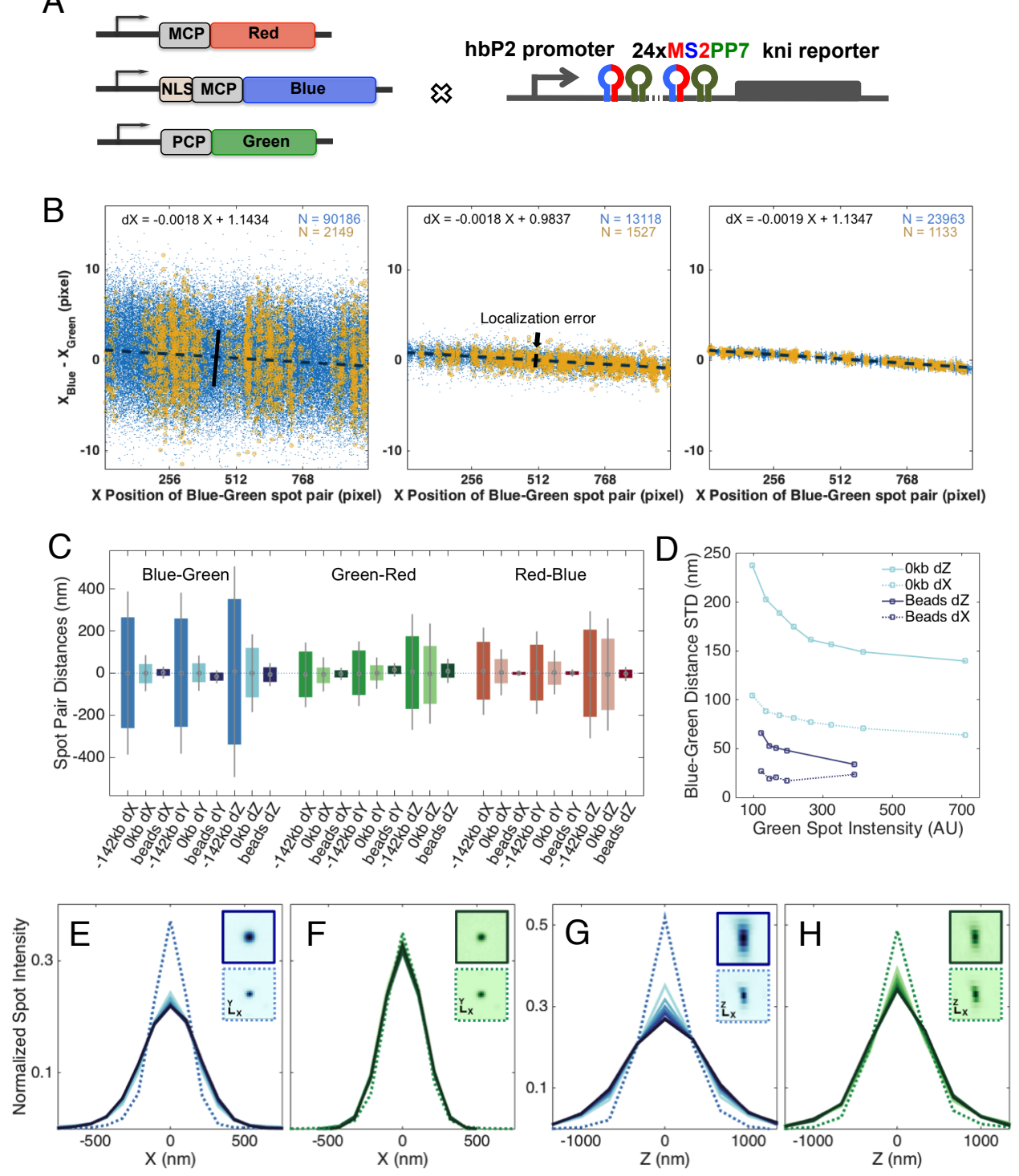

SUPP. FIG. 2: Spot localization precision and measurement error. (A) Genetic design of a transgene that co-localizes all three reporter systems. MS2 and PP7 stem loops are alternated and repeated 24 times. A kni reporter gene is driven by a hunchback P2 (hbP2) promoter, resulting in expression in all nuclei located in the anterior 10-45\% of the embryo. (B) A sample data set accompanying the methods for chromatic aberration correction and measurement error determination. Panels show the linear distance (along x-coordinate only) for each blue-green spot pair as a function of the pairs x-position for embryos carrying the parS-homie-lacZ transgene (left), embryos carrying the three-color co-localization transgene from (A) (middle), and TetraSpec beads (right), respectively. Blue data points are for all spot pairs at all time frames for all embryos analyzed. Yellow data points are from one of the embryos (or one set of experiment for the beads). Linear fits in each panel report on the chromatic aberrations between blue and green spots in the x-direction. As slopes and intercepts for the different samples show no significant differences, chromatic aberrations can be corrected for each individual embryo data set internally. (C) Summary of the distributions of spot pair distances (after chromatic aberration correction) for the three configurations in (B). Each direction ( $\mathrm{x}, \mathrm{y}$, and $\mathrm{z}$ ) is shown for each color combination. For example, for the blue-green (MS2-parS) distances on the x-direction the STD of the parS-homie-lacZ transgene (labeled -142kb) corresponds to the solid black bar shown in the left panel of (B). Spot localization errors are estimated from the STDs measured with the three-color co-localization control embryos (labeled 0kb). Solid lines: standard deviation (STD); bars: $25 \%-75 \%$ quantiles. (D) Dependence of localization precision on signal intensities. Since localization precision scales with the square root of the number of photons, we directly compare localization errors from the three-color co-localization control embryos with the errors measured from immobilized beads of the same fluorescent intensity values. Panel shows that the differences in the localization errors between embryos and beads are not due to difference in photon counts, and thus about $2 / 3$ of that localization error is due to motion blurring of the moving spot during acquisition. The remaining $1 / 3$ (i.e. error obtained from immobile beads) stems from optical measurement noise and the analysis pipeline. (E-H) Optical characterization of transcription and parS spots. For each fluorescent channel, all identified spots are classified into eight groups according to their intensities. An average spot for each group is created by aligning all spots so that the brightest pixels are at the center of a $25 \times 25 \times 13$ voxel region of interest $(\mathrm{ROI})$ and taking the average intensity per voxel in that region over all spots. The intensity profiles along the X- (E and $\mathrm{F}$ ) and Z-cross-sections ( $\mathrm{G}$ and $\mathrm{H}$ ) for the blue MS2 average spot (E and $\mathrm{G}$ ) and green parS average spot (F and H) are plotted (darker curves represent brighter spots). Dashed lines are from equivalent measurements of TetraSpec beads. Images of the average spots for the brightest blue (or green) MS2 spots (upper) and for the beads (lower) are shown as panel insets. 
bioRxiv preprint doi: https://doi.org/10.1101/099523; this version posted January 31, 2017. The copyright holder for this preprint (which was not certified by peer review) is the author/funder, who has granted bioRxiv a license to display the preprint in perpetuity. It is made available under aCC-BY-NC 4.0 International license.

A

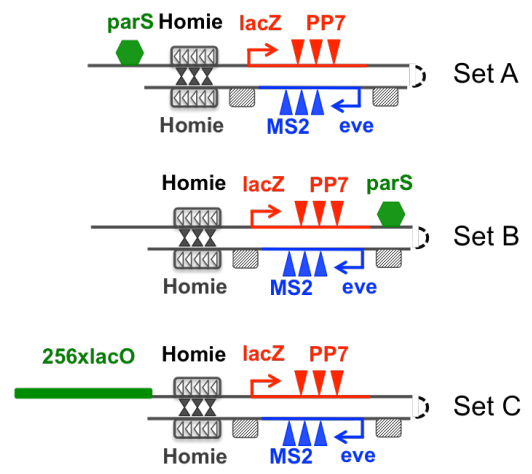

B

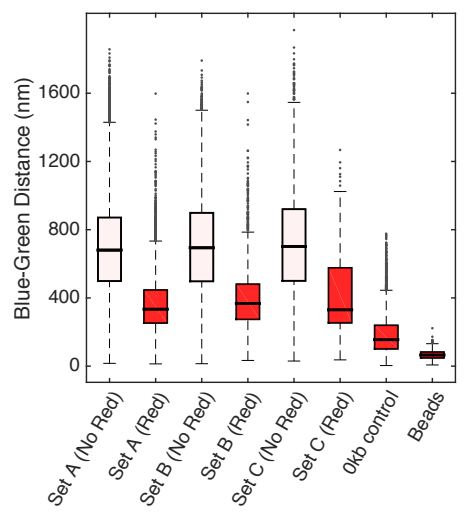

C

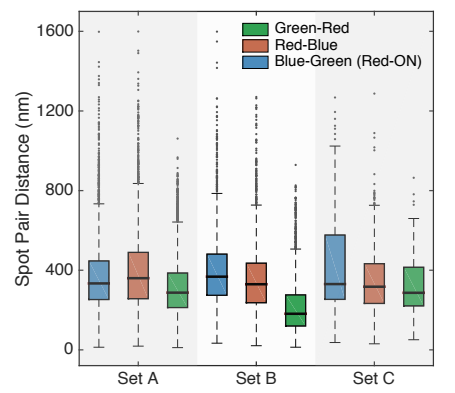

D
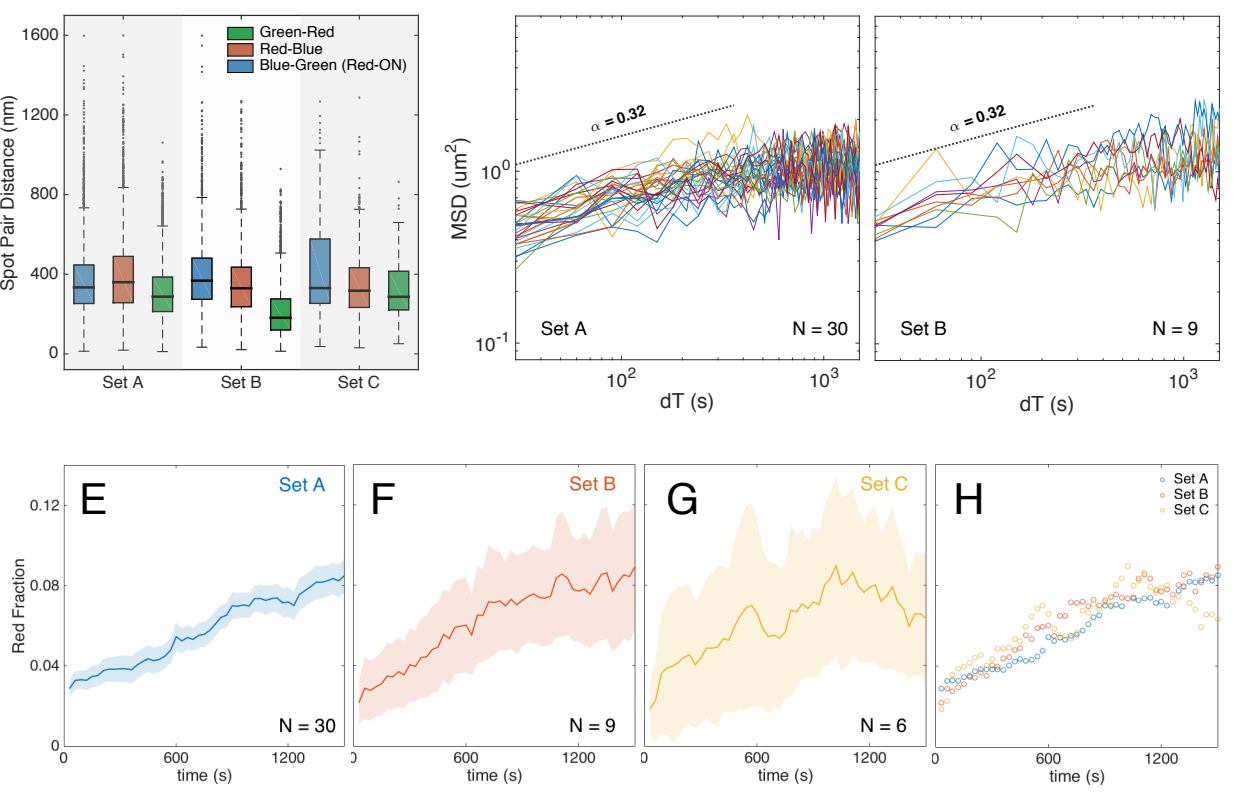

SUPP. FIG. 3: Different genomic labeling approaches report on similar chromatin dynamics and transcription kinetics. (A) Three methods of labeling genomic loci. (B) The measured Blue-Green (MS2-parS) distances are not sensitive to labeling approaches. Boxplot showing distributions of the instantaneous distance between spot pairs in the same nuclei. Distances shown are after chromatic aberration corrections. For all three genomic settings, the MS2-parS (blue-green) distances show no significant differences. This is observed regardless the absence (No Red) or presence (Red) of lacZ activity. The whiskers extend to 1.5 times the inter quantile range from the $25 \%$ and the $75 \%$ quantiles. (C) The distances between spot pairs reflect their genomic arrangement. Distributions of the instantaneous distance between spot pairs are plotted. Box and whiskers as in (B). Distances shown are after chromatic aberration corrections. Notice that the parS-PP7 (green-red) distance is significantly shorter when the parS tag is located at the 3 side of the lacZ repoter. (D) Mean square displacement (MSD) plots for Set A and Set B. Each MSD trace is a result of population ensemble of all nuclei in a single embryo (embryo-averaged MSD, see Methods). Results from the two genomic settings display sub-diffusive characteristics with a scaling power of 0.32 and their anomalous diffusion coefficients show no significant difference. (E-H) Transcriptional activation of lacZ-PP7 is not affected by labeling approaches. The fraction of eve-expressing nuclei that also contain active lacZ-PP7 is plotted as a function of time for three genomic settings. Apparently, the presence or locations of parS tags do not interfere with enhancer actions and transcriptional activation. 
bioRxiv preprint doi: https://doi.org/10.1101/099523; this version posted January 31, 2017. The copyright holder for this preprint (which was not certified by peer review) is the author/funder, who has granted bioRxiv a license to display the preprint in perpetuity. It is made available under

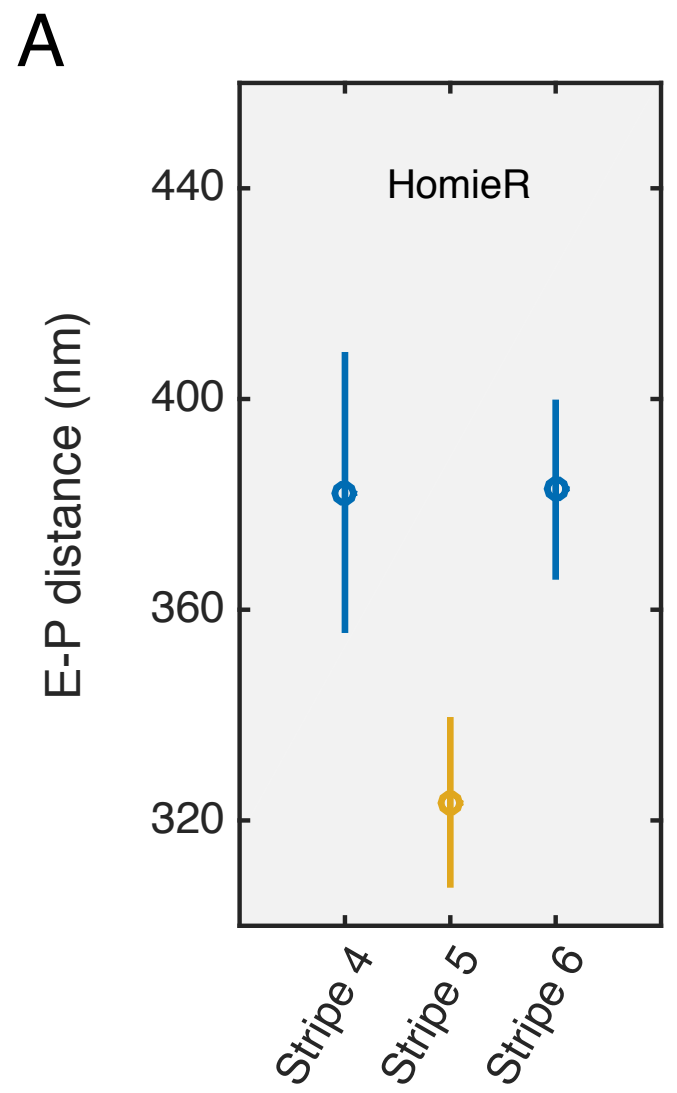

SUPP. FIG. 4: Stripe-specific topological difference for the parS-homieR-lacZ transgene. (A) RMS distance between blue (MS2) and green (parS) DNA foci (E-P distance) for all nuclei in which homie pairs with the reversed homie (homieR) sequence. RMS distances are calculated for individual eve stripes. 